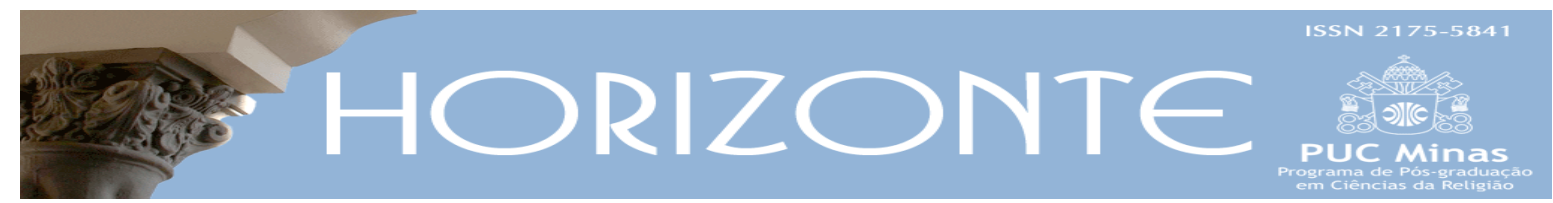

Dossiê: Ordens Religiosas Medievais: Poder e Sociedade - Articolo orginale (-) 0

DOI - 10.5752/P.2175-5841.2017v15n48p1299

\title{
La legittimità del potere ed il suo esercizio. Elementi comparativi nella testualità politica francescana del XIV secolo.
}

The legitimacy of power and its exercise.

Comparative elements in the fourteenth-century Franciscan political textuality.

Paolo Evangelisti *

\begin{abstract}
Riassunto
L'esperienza medievale dei Minori è caratterizzata dalla decisione di svolgere un ruolo attivo nelle realtà sociali nelle quali l'Ordine si inserisce. In questo quadro la consapevolezza teorico-politica francescana matura sino a cimentarsi con la questione dei requisiti che, da soli, possono legittimare l'autorità politica.Se in Scoto la legittimità deriva in via esclusiva dall'espressione del consenso di coloro che volontariamente scelgono di conferire quel potere ad un'autorità, se in Ockham la legittimità del potere è condizionata da un duplice principio di circostanzialità e di efficienza funzionale, in Eiximenis la questione della legittimità è sottoposta a un duplice vaglio.L'agire politico dell'autorità politica non può mai essere considerato un esercizio della plenitudo potestatis in ragione di un principio costituzionale: il reggitore di una comunità deriva il suo potere e la sua autorità non solo dal consenso ma da un patto convenuto che deve avere per oggetto esplicito gli ambiti ed i fini della sua azione. Il secondo vaglio è inerente ad una condizione coessenziale di legittimità: il potere ha una sua motivazione strettamente funzionale. L'autorità politica deve garantire, classicamente, pace e giustizia, ma anche farsi parte attiva di un processo volto al "millor estament de la cosa pública", al miglioramento economico dei soggetti che costituiscono la comunità politica, ma anche di coloro che potranno entrarne a far parte attraverso precisi e ben delimitati percorsi inclusivi.
\end{abstract}

Parole Chiave: Francescani; Legittimazione Del Potere; Teoria Politica; Analisi Istituzionale.

\begin{abstract}
The medieval experience of the Franciscans is marked by the decision of acting in different societies. In this context the awareness of their presence bring them to focus on the requirements that, by themselves, can legitimize the political authority. If in Scoto legitimacy is exclusively founding by the consensus of the people who voluntarily choose to confer its power to an authority, if in Ockham the legitimacy of power is conditioned by the double principle of circumnstantiality and functional efficency, in Eiximenis the basis of the authority is submit to a double temperament. First of all the authority can never be considered as an exercise of plenitudo potestatis due to a constitutional principle: the ruler of a community derives his power not only from the consensus but from an agreed pact in which is clearly fixed circles and ends of his action. The second one regards the co-essential condition of the legitimacy: the power of the ruler has a strictly functional justification. This kind of authority must ensure not only the classicals peace and justice, it has to become an engine of the process looking for the "millor estament de la cosa pública", for the economic emprovement of the subjects belonging to the political communitiy, but also of those who could enter in it by a specific and very well circunscribed pathways of integration.
\end{abstract}

Key-Words: Franciscans; Legitimisation; Political theory; Institutional analysis.

Articolo presentato il 6 dicembre 2017 e approvato il 12 dicembre 2017.

* Professore di Facoltà/Qualifica: Scuola Superiore di Studi Medievali e Francescani/Invitato. Laureato in storia all'Università di Trieste. Cultore della materia in storia medievale presso I'Università di Trieste. Paese di origene: Italia. E-mail: pevangelisti2000@yahoo.it

Horizonte, Belo Horizonte, v. 15, n. 48, p. 1299-1323, out./dez. 2017 - ISSN 2175-5841 


\section{Introduzione}

L’ordine mendicante francescano sviluppa, sin dai suoi primi passi, un intenso rapporto con le città, con il loro tessuto civile, sociale, economico e istituzionale. Numerosi indici così come una vasta tipologia di fonti ci offrono la possibilità di misurare il radicamento e l'espansione di questo rapporto che assume una dimensione compiutamente europea già nella seconda metà del XIII secolo. Basti por mente al ruolo svolto dalle chiese francescane che divengono autentici poli di attrazione di segmenti organizzati e non organizzati della società cittadina così come si può rinviare ai dati che emergono dalla localizzazione dei conventi, dalle modalità con cui i Minori ottengono spazi edificabili e finanziamenti dedicati. In quel medesimo torno di tempo le scritture notarili e altre fonti scritte ci raccontano dei lasciti testamentari nella duplice forma di legati diretti e di affidamento dell'esecuzione dei diversi lasciti conferiti ai conventi e a singoli francescani da parte di cives di diverso peso sociale. Non meno rilevante, anche in questa direzione, si sta rivelando l'analisi dei libri contabili dei conventi, tra gli esempi più ben studiati: Padova (1263-1302), Avignone (1379-1458) (LENOBLE 2012) e Gilet nel regno di Valencia (1402-1487) (MANCINELLI 2017). Ma la presenza dei Minori nel tessuto civile, politico ed economico delle città e degli stati territoriali si connota anche per la frequente opera di consiglio, di collaborazione, di attiva interlocuzione prestata alle istituzioni che amministrano e governano porzioni significative dell'Europa di XIII-XIV secolo. Un'attività che l'Ordine intende in qualche modo disciplinare come ci rivela una disposizione che si legge nelle Costituzioni narbonesi approvate nel 1260, (cap. VI.7), reiterata almeno sino al 1325. In proposito sarà sufficiente ricordare che tra il 1276 ed il 1458 nelle istituzioni della sola corona aragonese prestano la loro opera oltre centotrenta frati Minori che ricoprono ufficialmente incarichi di rilevanza politica e consiliativa. Sono dati che non tengono conto delle cariche vescovili e della specifica realtà del regno di Maiorca che vedrà sul trono, in qualità di reggente, un discepolo di Angelo Clareno: Filippo di Maiorca (1325-1329) (EVANGELISTI 2006, 2007). 


\section{Il potere sotto la lente dei Minori: il contributo decisivo del Doctor Subtilis}

In uno dei capitoli introduttivi della sua più importante opera politica Francesc Eiximenis, un francescano attivo nell'ultimo quarto del XIV secolo, riflettendo sulla natura e le funzioni della civitas ritiene necessario richiamare l'esempio del fondatore del suo Ordine quando, all'inizio della sua nuova vita, si chiede dove sia più utile svolgere la propria azione, quale sia il migliore contesto dove poter agire insieme ai suoi compagni che lo seguirono in quella scelta di conversio e di povertà volontaria:

Nos ... sabem que sent Francesch demanà a Déu si sos frares estarien en los deserts o en les ciutats. E estech-li respost que en les ciutats e viles per tal que per lur preÿcació tirassen les anime a Déu ...”. E' quindi, prosegue il testo, "voluntat de Déu" che i Minori "estiguen en les ciutats per informar los ciutadans a bé viure [...] (EIXIMENIS, 2005, cap. 25, p. 25).

Fin dall'inizio della sua opera, costruita come un testo di pedagogia e dottrina politica, il francescano catalano mette così insieme il valore della polis, luogo per eccellenza delle relazioni civili ed economiche e il valore dei Minori, la loro fondamentale funzione nella città.

E' Dio stesso a chiedere loro di non stare in un monastero cinto di mura lontano dal mondo, ma di stare nella città, e questo messaggio fondamentale lo consegna allo stesso Fondatore dell'Ordine dei poveri volontari.

Si potrebbe dire che con questa operazione di recupero di un frammento biografico di Francesco Eiximenis consegua efficacemente un duplice obiettivo: nobilitare il valore del vivere civile e consacrare la funzione politica, pedagogica dei francescani chiamati ad agire dentro la civitas.

Il frammento che sta dinanzi a noi segnala però, in maniera più generale, l'esistenza di una particolare sensibilità ed attenzione dei Minori nei confronti di quell'ampia sfera del vivere sociale che si svolge dentro la città e nelle sue istituzioni. Una sensibilità che, considerate in una prospettiva storica, si trasforma e si qualifica 
in un'autentica capacità di osservare queste relazioni, di analizzarle, e, su questa base, fatta di attenzione e di empiricità, produrre codici valoriali, autentici elementi di dottrina politica, ma anche concrete linee progettuali di governo.

All'interno di quest'ampia attenzione francescana per le dinamiche della vita civile, per le forme ed i contenuti del governo delle società nelle quali operano, riveste particolare interesse il discorso sviluppato dai Minori, sin dall'inizio del secolo XIV, su una delle questioni che resteranno centrali nella storia delle dottrine politiche sino ai nostri giorni.

Si tratta dell'analisi che pone in primo piano la questione non delle forme o dell'eticità del potere, griglie intellettuali attraverso le quali sino ad allora l'intera riflessione politica svolgeva il proprio ragionamento circa il fondamento stesso del potere, ma dei requisiti che, da soli, possono legittimare l'autorità politica, dunque validare il potere ed il suo esercizio. Sono noti a tutti i nomi di due magistri francescani che producono queste analisi: il Doctor Subtilis, Duns Scoto, e il Doctor Invincibilis, Guglielmo di Ockham.

E'un dato già ampiamente rilevato dalla storiografia, ma è utile ricordarlo in questa sede. Ancorché nelle prossime pagine l'analisi si concentrerà sui testi di Scoto e su quelli di un suo allievo catalano è infatti indispensabile richiamare l'attenzione su un tratto comune che unisce i due magistri inglesi: il nesso inscindibile tra la loro identità ed appartenenza minoritica e la profonda capacità autoptica di entrambi esercitatasi sul potere come forma del dominio degli uomini sugli uomini (LAMBERTINI, 2000).

Questo per almeno due ordini di ragioni che rivestono una precisa valenza dottrinaria.

La prima è immediatamente leggibile nei loro testi: entrambi i magistri giungono a riflettere sui fondamenti primi di legittimità e di funzionalità del potere interrogandosi sui fondamenti della proprietà personale, sulle origini di uno ius proprietario che è coessenziale alla stessa nascita del dominium. 
La seconda è altrettanto evidente e strettamente connessa alla prima: se proprietà, dominium, uso dei beni, uso del potere possono essere interrogati insieme, fanno problema, ciò si verifica perché il problema viene percepito ed affrontato come quesito radicale da chi è, per propria scelta, povero volontario, vale a dire soggetto che si è impegnato a non possedere alcunché, a rinunciare ad ogni diritto sui beni, ma ad usare con consapevolezza le risorse del mondo. Il francescano è dunque obbligato, nel momento stesso in cui entra nella vita proposta dalla Regola di Francesco, ad interrogarsi su ogni forma di dominium, su ogni forma di potere e sulle condizioni in cui questi dominii si esercitano, possono o non possono essere usati, e da chi. Una delle conferme più risalenti in questa direzione viene dalla produzione intellettuale del più noto francescano provenzale del XIII secolo. Pietro di Giovanni Olivi, solo qualche decennio dopo la stesura della Regola minoritica, affrontò infatti ex professo questo tema in una dissertazione significativamente intitolata Quid ponat ius vel dominium (PIETRO DI GIOVANNI OLIVI, 1945).

Questa questione, che si pone sotto il profilo normativo e più in generale ermeneutico-filosofico ad ogni frate che entri nell'Ordine, va quindi riconosciuta come autentica determinante storica della riflessione politica sviluppata dai francescani sul potere. E, in questo senso, attesi i risultati politologico-dottrinari che tale riflessione sviluppa, non si potrà non prendere atto che il formarsi del linguaggio politico medievale ha radici e spinte che non possono esaurirsi in quella "riscoperta di Aristotele" che ancora oggi molti tra gli storici della filosofia e del pensiero politico ritengono essere la vera ed unica conditio sine qua non della modernizzazione, o, per altri versi, della "secolarizzazione" intesa in chiave "evolutiva" del pensiero politico medievale ${ }^{1}$. Ciò non solo per quanto si verrà esponendo in queste pagine, e per quanto, ben più autorevolmente, hanno dimostrato decenni di produzione storiografica in particolare italiana e tedesca sulle matrici francescane della riflessione politica, ma per altri ed ulteriori fattori che non possono essere sottostimati proprio nella prospettiva della storia del pensiero politico medievale e

${ }^{1}$ Utili considerazioni in proposito si leggono in Carl Nederman (NEDERMAN2009, p. 3- 12). 
moderno. Si pensi in particolare agli apporti che, oltre alla specifica testualità francescana che sarà qui esaminata, vengono da quei linguaggi giuridici e da quelle codificazioni normative messe in forma proprio in età medievale da concili, sinodi e concordanze canonistiche, da quei linguaggi patristici che, per il tramite dell'esegesi biblica e della cura pastorale, producono analisi politica ed economica, costruiscono etiche e dottrine che ritroviamo pienamente in testi del Bassomedioevo, ma anche nei testi sacri fondanti la dottrina politica moderna: basti pensare ai numerosi debiti linguistici e concettuali rilevabili nelle opere di Marsilio, Hobbes e Locke. Ci si riferisce in particolare a quell'enorme giacimento di codificazioni politiche messe in campo nell'età patristica e medievale studiate da coloro che hanno riflettuto sulla teologia politica e sulla teologia economica sin dagli studi pioneristici di Post e Kantorowicz, per giungere, dopo Capitani e Todeschini (CAPITANI 1990; TODESCHINI 1994) a Giorgio Agamben (AGAMBEN 2007; AGAMBEN, 2011²), Jan Assmann (ASSMANN 2000), Roberto Esposito(ESPOSITO 2013) e Valentina Toneatto (TONEATTO, 2012), senza dimenticare l'apporto venuto dai testi di Carl Schmitt (SCHMITT, 1972;SCHMITT, 2009).

Andiamo dunque al cuore della questione politico - dottrinaria che vogliamo esaminare.

Il pensiero politico che emerge da una pluralità di testi del secolo che vede la nascita e la straordinaria diffusione degli Ordini Mendicanti e del francescanesimo in particolare registra, quanto alla riflessione sull'origine del potere, un elemento di persistente assenza. Chi si interroga su tale questione, da Tommaso d'Aquino a Tolomeo da Lucca, da Egidio Romano a Giacomo da Viterbo, rimane ancorato ad una riflessione che guarda al potere secondo il modello della Politica di Aristotele. Vale a dire guarda al potere nelle sue forme di manifestazione e di esercizio, chiedendosi quale sia la migliore e/o la più utile. Guarda inoltre al potere, quanto alla sua autorevolezza ed al suo fondamento, come ad un’istituzione che in ogni caso

${ }^{2} \mathrm{Per}$ il secondo testo di Agamben rinvio anche alle importanti osservazioni proposte da Clement Lenoble e Valentina Toneatto (LENOBLE\&TONEATTO, 2013). 
deriva da Dio e dal pontefice, ovvero da Dio e dall'imperatore o dal monarca, o ancora da Dio e dal trasferimento del potere del popolo al monarca, secondo una scala assai ampia di differenziazioni.

La prospettiva cambia in maniera radicale se ci volgiamo alla testualità politica francescana, a partire da ciò che scrive Duns Scoto nel suo testo sul potere più noto: l'articolo 1 della quaestio 2 contenuta nella distinctio 15, Ordinatio IV, del suo Commento alle Sentenze, un testo frutto delle sue lezioni tenute tra Oxford e Parigi, databile al 1303-1304. Scoto giunge alla questione del potere politico per una via che appare decisiva,ancorché né scontata né, tantomeno, tradizionalmente percorsa, vale a dire come esito della sua riflessione circa il dovere del buon cristiano alla restitutio dei beni illegalmente sottratti e, risalendo a monte del problema, riflettendo sull'origine e la validità della proprietà privata sui beni

Se devo sapere se sono tenuto a restituire i beni sottratti a qualcuno illegalmente devo sapere innanzitutto che cosa si può definire come bene di proprietà di qualcuno e, se devo definire la proprietà, devo sapere quale sia la sua origine e chi possa stabilire che una cosa appartiene a qualcuno" (Ordinatio IV, distinctio 15, quaestio 2, art. II).

E' da questo punto che egli muove per sostenere che la "distinzione delle proprietà" non è avvenuta secondo una legge divina o naturale, ma secondo una legge interamente umana, cioè "secondo una legge positiva”. Il dominium degli uomini sulle cose richiede quindi e porta Scoto ad interrogarsi sul dominium degli uomini in sé. E si noti che qui il potere di distinguere e governare le proprietà non viene interrogato nella sua epifania, nella sua forma: monarchia, aristocrazia, oligarchia e così via, ma secondo la sua natura essenziale che il francescano coglie come natura esclusivamente giuridica. Il suo ragionamento, infatti, prosegue sostenendo che "per verificare" se la "distinzione" delle proprietà "sia giusta, sarà necessario considerare a quali condizioni tale legge positiva sia giusta”. Se dunque il problema è quello della giustezza della legge positiva quale unico e primo fondamento del dominium sulle cose è indispensabile andare alla fonte di questa 
giustizia della legge umana, ovvero stabilire chi possa porla. Con le parole di Scoto: "la legge positiva richiede l'autorità" e quindi la conclusione è "che la legge positiva giusta richiede il legislatore", un legislatore dotato di saggezza ed autorità. Si vede qui come la coerenza logico-argomentativa del Doctor Subtilis sia rigorosa e precisa. La questione è posta come questione del tutto impersonale, il quid è il rapporto tra legge positiva giusta e autorità dotata di tutti i requisiti per porla. Questa autorità, mantenendo il profilo di attenzione alla sostanza giuridica e politica, viene chiamata il legislatore.

Quella percorsa da Scoto per costruire una dottrina politica è dunque un'altra via non solo filosofica, ma linguistica ed argomentativa. La questione del potere non viene affrontata nella sua datità, nel suo essere frutto di una serie di condizioni derivanti e derivate. La questione della sua origine viene indagata direttamente ed esattamente come un problema che riguarda la sua esclusiva legittimità.

Se la legge positiva per essere giusta, dunque per essere valida, cogente e applicabile, deve derivare dall'autorità, allora si deve verificare, si noti il verbo, se quell'autorità sia legittima, possa cioè legiferare, e porre dunque norme valide e vincolanti. Per Scoto la legittimità consiste esattamente in questo: per avere una legge giusta è “evidentemente necessario" avere "l'autorità giusta”. A differenza della classica posizione dei commentatori della Politica aristotelica, dei testi stessi realizzati per l'educazione del principe, solo successivamente Scoto giunge al problema delle forme possibili dell'autorità che viene quindi affrontato come un problema secondario e, soprattutto, non dirimente.

La qualità e la giustezza dell'autorità politica non si misurano né si esauriscono nella forma istituzionale dell'autorità, ma la questione è a monte: riguarda esattamente il suo costituirsi, atto logico e giuridico che precede la veste nella quale il legislatore prenderà forma. Terminologicamente questa insistenza sulla nozione di autorità politica come soggetto impersonale, come soggetto qualificato solo dal suo potere legiferante, trova evidentemente un chiaro nesso con il metodo e con il linguaggio che Marsilio adotterà un ventennio dopo nelle sue opera 
politiche, nel suo ripensamento radicale circa il fondamento dell'autorità politica come autorità politica umana. Il ripensamento posto sin dall'avvio del primo discorso del suo Defensor pacis, a partire da ciò che si sostiene nel capitolo XII (MARSILIO DA PADOVA, 1975²; p. 170- 250; BRIGUGLIA, 2013, p. 63-116 e p. 171206; DOLCINI, 1988, p. XX).

Scoto afferma quindi, quanto al problema secondario della forma, che l'autorità legittima, il "potere pubblico" che si esercita come politico, cioè non domestico, può risiedere in una persona sola o in una comunità. Ma, a conferma della secondarietà di questo problema, il suo ragionamento prosegue nell'affermare che "quanto all'autorità politica, sia che risieda in una persona sola sia che risieda in una comunità" essa"può essere giusta” in base a due soli elementi: "in base al comune consenso e all'elezione della comunità stessa" (Ordinatio $I V$, d. 15, q. 2, n. 7) 3 .

Si tratta, come è evidente, di un tipo di teoria fondazionale del potere che assume una rilevanza sotto molteplici punti di vista.

In primo luogo perché essa viene fatta derivare da una questione di ordine puramente economico: il potere nasce, si costituisce in autorità, per governare e legiferare sulle proprietà che ogni uomo può possedere. E' un'autorità politica che veglia sulle proprietà che ogni uomo deve veder tutelate rispetto agli altri uomini. Il fondamento di questo potere autorevole ha nella legittimità il suo fattore irrinunciabile ed esclusivo ed, inoltre, i caratteri ed i requisiti di questa legittimità politica e giuridica sono a loro volta esclusivamente umani. Scoto, dunque, non contribuisce solamente a ridefinire il potere e l'autorità attraverso la legittimità, non contribuisce solo ad affermare che questa legittimità è fondata su requisiti che risiedono interamente nella dimensione umana, ma, con questi nuovi paradigmi, conferisce una forza nuova all'autonomia del potere temporale, al potere e all'autorità politica tout court.

\footnotetext{
3“... Utpote, si ad civitatem aliquam aedificandam vel inhabitandam concurrerunt extranei aliqui, videntes se non posse bene regi sine aliqua auctoritate poterat consentire, ut vel uni personae vel communitati committerent illam communitatem: et uni personae vel pro se tantum - et successor eligeretur sicut ipse - vel pro se et tota sua posteritate"; (DUNS SCOTO,2001, p. 34).
} 
Dentro a questo rinnovamento di ordine teorico-politico c'è, inoltre, l'emersione del fattore costituzionale. Agli occhi di Scoto un'autorità politica pubblica è legittima, e dunque soggetto valido di potere, perché acquisisce questa validità, che è giustezza e giustizia, sulla base esclusiva del consenso che per via deliberativa e volontaria viene ad essa accordato da coloro che, avendo comunemente constatato "di non poter convivere bene senza la presenza di alcuna autorità", ritengono di "trovare un accordo comune per conferire a un'unica persona oppure a un gruppo il potere su quella comunità". "In questo modo", conclude perentoriamente e assertivamente Scoto, “abbiamo assolutamente tutto quantosi richiede per porre in essere una legge positiva, a opera di chi è saggio oppure si avvale di consiglieri saggi, ed è dotato di una giusta autorità secondo le forme [qui] esposte"4.Come si vede la questione della forma perfetta del potere, cara a tutti i pensatori coevi al francescano, non viene affrontata, qui anzi non viene neppure nominata. Per Scoto la questione del potere si colloca a monte: esso può essere indagato e legittimato solo se si comprende che tale riflessione teoretica deve rimanere estranea ad ogni personificazione e solo se si comprende che la sua unica concretezza è data dalla ragione e dal diritto. Scoto coglie qui l'elemento cruciale, si potrebbe dire ontologico dell'autorità politica poiché essa sorge, viene individuata, solo in quanto necessaria per porre la legge, solo in quanto necessaria alla comunità che vuole averla per affidarle un compito ben preciso: opporre alla bia una dike che sia legittimamente fondata, autorevole e dunque effettivamente ed efficacemente cogente.

Un aspetto non secondario della filosofia politica messa in forma da questa argomentazione è quello che può essere definito come l'origine positiva del potere politico pubblico. In effetti, a differenza di molti pensatori che troveranno in età moderna il loro esponente di riferimento in Hobbes, in Scoto - ancor più che in Ockham e in altri pensatori politici francescani -, l'origine dell'autorità, la cogenza del potere non nasce dalla paura, da un bisogno di sicurezza posto in sé, quasi

4"Ergo habemus complete quomodo poterat condi lex positiva iusta, quia ab habente prudentiam in se vel consiliarii suis, et cum hoc habente auctoritaem iustam aliquo modo dictorum modorum in ista conclusione"; (DUNS SCOTO2001, p. 34, corsivi nostri). 
scaturente da un terrore dell'altro, ma da una assai più piana necessità di regolamentazione, di un ordinato vivere al quale la comunità acconsente liberamente, e soprattutto, per comune e volontaria deliberazione: "videntes se non posse bene regi", i membri di quella communitas stabiliscono di "concorditer consentire ut vel uni personae vel communitati committerent illam communitatem” (DUNS SCOTO, 2001, p. 34, corsivi nostri).

In questa teoria fondazionale del potere c'è quindi un duplice portato dottrinario.

Da un lato vi è la radicale presa di posizione sulla legittimità e sul consenso che configurano una concezione del formarsi del potere come principio ascendente rispetto al soggetto che poi riceverà l'autorità per poterlo esercitare.

Dall'altro vi è una positività strettamente connessa alla dimensione umana della sua genesi, così come della funzione assegnata al legislatore ed alle norme giuste che da esso possono venir poste. Il potere in quanto tale viene quindi costituito ed è autorizzato a dispiegarsi, ad agire, “per poter convivere bene”, per poter ben governarsi: il testo latino dice, concretamente e tecnicamente, per poter "bene regere". Scoto conferisce così all'autorità ed al potere stesso una intensione e una prospettiva d'azione integralmente destinate al benessere comunitario, allo sviluppo della res publica. Tant'è che la teoria scotiana della legittimazione dell'autorità politica afferma che la comunità potrebbe anche governarsi da sé e non conferire necessariamente il potere ad una o più persone:

si ad civitatem aliquam aedificandam vel inhabitandam concurrerunt extranei aliqui, videntes se non posse bene regi sine aliqua auctoritate poterant concorditer consentire ut vel uni personae vel communitati committerent illam communitatem (DUNS SCOTO, 2001, p. 34, corsivi nostri).

Nella ratio originaria, che esplica la funzione dell'autorità e del potere umani, a prevalere è questo elemento volitivo e positivamente comunitario non quello della sicurezza, intesa come difesa dall'altro. E si noterà che questo bene, questo bene regere, non è qui inteso come un bonum di natura morale, ma è un bonum che si 
qualifica come benessere, come qualità di vita comunitaria, un bonum interamente umano e politico che non può essere confuso o sovrapposto con l'ipostasi del bonum commune. Basterebbe scorrere il prosieguo di questa stessa quaestio per scorgervi le applicazioni e le implementazioni che Scoto dà a tale bene comunitario. Nell'articolo II della quaestiol'attenzione del francescano si concentra infatti sulla necessità di potenziare gli scambi economici e di migliorare l'organizzazione commerciale sino al punto da asserire che la bontà del legislatore si misura sulla sua capacità di attrarre e favorire all’interno della res publica la presenza di abili e competenti mercatores i quali debbono trovare in quella res publica condizioni civili ma anche adeguati compensi per la loro professione (DUNS SCOTO, 2001, p. 56$60)^{5}$.

Va rilevato infine un fatto di notevole valenza dottrinaria ovvero che, proprio nell'articolo dedicato alle problematiche economiche e mercantili, il Doctor Subtilis torni a riflettere sul valore e la funzione del consenso politico.

In questa sezione della quaestio egli ribadisce infatti il ruolo fondativo del consenso individuale come elemento indispensabile sia nell'istituirsi della comunità sia nell'emanazione di una legge che solo così può definirsi giusta. E' da questa premessa costituzionale che è possibile definire la translatio dominii, ovvero una legittima modalità di trasferimento delle proprietà che può avvenire non solo tra privati, ma anche secondo due ulteriori modalità: per mezzo dell'autorità pubblica $\mathrm{o}$ per legge. Nel caso della translatio dominii auctoritate publica la facoltà, risiedente nella comunità, di trasferire a qualcuno un bene che appartiene alla comunità nella sua interezza deriva precisamente dal consenso che regge e valida l'istituzione della res publica.

Con questo passaggio si registra quindi una saldatura di natura non retorica, ma fondata su una razionalità ed una sistematicità proprie dell'argomentazione scotiana, tra la teoria del potere politico legittimo, originato esclusivamente dal

${ }^{5} \mathrm{E}^{\prime}$ una riflessione che trova un suo importante antecedente nel De empcionibus et vendicionibus di Pietro di Giovanni Olivi (TODESCHINI, 1980, p. 63- 64; TODESCHINI, 2002, p. 350, n. 73). Sull'intera questione un'utile recente messa a punto si legge in un saggio di Igor Mineo (MINEO, 2014). 
consenso, e la analisi della iustitia nelle transazioni economiche. La legittimità del potere e quella delle transazioni economiche-vale a dire la iustitia del dominium degli uomini sugli uomini e quella del dominium degli uomini sulle cose- trovano così una radice comune e inderogabile: il consenso espresso delle volontà tra e dei soggetti interessati. La legge giusta, che deve essere emanata per disporre un trasferimento di una proprietà comune ad un soggetto, può prendere forma ed esprimere tale volontà giuridica e politica in quanto manifestazione di un soggetto collettivo che raccoglie e può utilizzare il consenso di tutti coloro che gliel'hanno conferito. E' dentro questo alveo politico e giuridico che viene a definirsi l'utilità stessa, in sintesi sostanziale l'economia di questo trasferimento di beni.

\section{La legittimazione dell'autorità e i poteri del governante sotto la lente di un allievo del Doctor Subtilis: Francesc Eiximenis}

Un discepolo di Scoto - la cui importanza è data in primo luogo dal suo essere stato contemporaneamente autore di opere politiche ed esponente di primo piano tra quei Minori che prestarono direttamente la propria competenza ai poteri civili e politici operanti nell'Europa medievale - Francesc Eiximenis, affronta la questione del potere e della legittimità dell'autorità politica con altrettanta determinazione dei suoi predecessori francescani, ma muovendo, in via prevalente, da altri luoghi classici della trattatistica due-trecentesca.

Il punto di attacco della sua riflessione su questo elemento centrale e fondativo di ogni teoria politica medievale e moderna non è posto nel momento originario storico-biblico in cui si definisce il nuovo statuto dell'uomo e le sue facoltà dopo la Caduta, in quella duplex potestas ricevuta nella condizione postlapsaria, facoltà concessa da Dio a ciascun uomo secondo la nota definizione e sistematizzazione offerta nel Breviloquium di Guglielmo di Ockham (OCKHAM, 1997, p. 180). Il frate catalano, che pure tratta e considera la questione della proprietà schierandosi apertamente per un diritto proprietario privato e dovutamente tutelato, ragiona sul fondamento del potere inserendo questo element 
nell'ambito della sua analisi volta a comprendere - e a rendere intellegibili ai suoi discenti, lettori della sua opera politica per eccellenza, il Dotzè del Crestià - le diverse ragioni del vivere comunitario. Vale a dire le cause determinanti che fanno della civitas la forma di elezione della vita sociale dell'uomo.

La polis è individuata dagli uomini come miglior forma di aggregazione civile e politica, a specificarlo è Eiximenis stesso, perché attraverso di essa si conseguono diversi obiettivi, tutti convergenti nella realizzazione della buona vita civile ${ }^{6}$.

In primis nella polis si combatte l'ignoranza che deriva dalla dimensione solitaria dell'uomo, secondariamente si realizza la conoscenza che è possibile solo in comunità, terza ratio e terzo obiettivo della civitas è evitare, sulla base delle conoscenze conseguite, tutti i comportamenti di errata gestione dei beni, delle risorse private e comuni, il quarto obiettivo è combattere gli uomini malvagi, vale a dire tutti coloro che non comprendono il senso ed il valore del vivere comunitario. Accanto a questi obiettivi, a questi autentici elementi fondanti della civitas, si recupera un luogo classico della Politica aristotelica per affermare un'ulteriore ragione costitutiva della civitas: la polis consente ai suoi cittadini di ben vivere. E' la quinta ratio posta da Eiximenis.

Questo ben vivere, che non riguarda tutti gli abitanti della città, ma i soli titolari dei diritti di cittadinanza, consiste in primo luogo nel "satisfer e provehir bestantment” ai bisogni, alle necessità dei consociati (EIXIMENIS, 2005, cap. 30, p. $64-65)^{7}$

Quindi anche il Gerundense ritiene, come Marsilio e a differenza di quanto sostengono più genericamente i commentatori della Politica attivi sin dalla seconda

\footnotetext{
${ }^{6}$ Le tredici ragioni che costituiscono in realtà i fini per i quali le civitates vengono edificate e vanno governate sono: "honor e glòria de Déu"; "per esquivar ignorància"; "per esquivar males cobejances"; "contrestar als mals homens e per defensor-se d'ells"; "provehir bestantment a les neçessitats dels hòmens"; "donar als hòmens honest pler e alegria"; la settima, ottava e nona ragione riguardano $\mathrm{i}$ motivi che determinano la fondazione materiale della civitas ovvero:"donare ai fondatori di città la loro giusta fama"; "celebrare la fama di qualche personalità o un'azione precisa compiuta da queste persone"; "l'individuazione della specialità di un luogo che si rivela particolarmente adatto alla edificazione di una realtà urbana"; le altre quattro ragioni fondanti e fondamentali sono le seguenti: "per servey especial de la cosa pública; "per necessitat de contractes"; per "regiment"; "per viure virtuosament". Si tratta di uno sviluppo politico-programmatico articolato lungo tutto il primo ed il secondo trattato del Dotzè del Crestiò (cap. 1- 356); per i capitoli 1212:EIXIMENIS, 2005, p. 1- 451.

${ }^{7}$ Su questo punto: (COSTA, 1999, I; EVANGELISTI, 2013;TODESCHINI,2007; TODESCHINI, 2013, p. 254- 277).
} 
metà del XIII secolo, che la polis non si debba dare come obiettivo la mera autosufficienza, il semplice soddisfacimento delle necessità primarie dei cives, ma debba garantire a tutti un ben vivere qualitativamente più elevato. Eiximenis, nel descrivere e qualificare questo obiettivo comunitario, antepone significativamente il verbo satisfer al verbo provehir. La descrizione della civitas come "congregacio sibi sufficientem" del testo latino con cui si viene a conoscere la Politica di Aristotele dopo il 1265, è interpretato dal frate di Girona, così come qualche anno prima avviene nel Defensor marsiliano, in modo più largo. In entrambi i testi la sufficienti avitae consiste nella piena soddisfazione dei bisogni che l'uomo elabora vivendo non nel deserto o in solitudine, ma nella civitas.

Eiximenis, esattamente a questo punto della sua analisi economico-politica condotta sulla città, inserisce un capitolo nel quale si affida a delle immagini, come le chiama lui stesso, dunque a icone dotate di una funzione legittimante per validare coloro che sono chiamati a governare la comunità politica.

Con questo preciso obiettivo vengono attivate nove icone, nove immagini di governanti capaci che incarnano al meglio questa funzione politica, scevra qui di ogni connotato connesso alle classiche virtù morali del buon reggitore.

Non è certo un caso, tenuto presente il contesto in cui è inserito questo capitolo, che la prima di tali icone sia quella di un mitico re di Tessaglia che, per primo, coniò denari d'oro e inventò i freni per i cavalli. Un re che dà così agli uomini civili gli strumenti per eccellenza dell'attività economica: la moneta più affidabile, misura degli scambi, e l'arte della cavalleria, capacità indispensabile per assicurare i movimenti delle merci, dunque la possibilità stessa del commercio dei beni.

E non è un caso che altre sette delle nove icone evocate da Eiximenis rappresentino altrettanti uomini di governo e di potere che hanno saputo individuare o favorire forme di aggregazione, strumenti od oggetti volti a realizzare al meglio gli scambi economici tra gli uomini civili.

Ancor più significativo appare il fatto che l'ottava di queste nove icone sia rappresentata da Forseo, unica figura regale attraverso la quale si affronta la 
questione dell'origine del potere in sé stesso, non semplicemente del potere funzionale a determinati obiettivi.

Si tratta di un dominium, di una potestas totalmente umani, analizzati da Eiximenis giungendo immediatamente al cuore del problema della legittimità dell'autorità politica:

hi avia ymatge de Forseo, rey de Bàctria, qui primer trobà corona, e sceptre e estament reyal per tal com ell primer féu ley, per manera de document general, que negun poble no elegís en son regiment negú al qual se donàs simplement, sinó que fos rector a temps cert, o a tots temps, ab certs patis que diguessen: "Axí.ns faràs, e axí.t farem; e si fas lo contrari, no.y volem per senyor. Sotsmetràs-te a nostra rahó e juy contra tu mateix en cert loch, axí com nós al teu quant siam trobats defallents (EIXIMENIS 2005, cap. 31, p. 67).

Il primo governante che individuò e seppe portare gli attributi del potere, corona e scettro, e si vide nel contempo riconosciuta la dignità regale, lo status di re, consegue quindi questa investitura attraverso un atto specifico: l'emanazione di una legge che ha evidentemente natura e forma costituzionale. Si noti che, nel costituirsi di questo potere, nella fondazione di questa autorità politica, Eiximenis non evoca alcun intervento esterno, sovraordinato, spirituale o divino. La legge che qualifica questo governante, il quale solo così può essere riconosciuto come tale, dotato di corona, scettro e status proprio, ha essa stessa non solo una centralità assoluta, ma anche una caratteristica indispensabile: è un documento scritto e deve avere valenza generale.

Accanto a questi due primi elementi va segnalato il terzo principio di validità della legge, vale a dire il suo contenuto prescrittivo, essendo essa dotata di un valore non solo ordinatorio ma perentorio: nessun popolo, nessuna comunità, può eleggere una persona alla quale viene conferito interamente e senza condizioni il potere di governo sulla comunità stessa. E' invece necessario che nell'atto di conferimento, nel documento costituente, vengano stabiliti due parametri inderogabili: la durata del trasferimento del potere e i contenuti di un accordo stipulato paritariamente tra governati e governante. Il rispetto dei contenuti di questo patto è garantito da un sistema di verifica e di eventuali sanzioni anch'esso assolutamente paritario quanto 
alla sua agibilità. E' infatti riconosciuto in primis alla comunità, ma anche al governante, il diritto di attivare questa verifica così come l'irrogazione di eventuali sanzioni per le inadempienze che dovessero essere registrate. Questo sistema di garanzie, che è parte integrante e norma di chiusura del testo costituente, prevede espressamente la decadenza dallo status di governante-reggitore qualora la comunità constati un'infrazione dei patti reciprocamente assunti e convenuti. Nello stesso tempo l'autorità politica che riceve il potere e la sua legittimazione in questa forma accetta di essere sottomessa alla ratio ed al giudizio della comunità, dunque accetta non solo la superiorità della legge in generale ma espressamente questa norma che ha natura e forza costituzionale ed è, insieme, cogente, valida ed efficace. D'altro canto la comunità stessa si impegna, in maniera simmetrica, a riconoscere ed accettare la verifica ed il giudizio del governante su eventuali inadempienze messe in atto dalla comunità stessa, ivi comprese le eventuali conseguenze da esse derivanti. Al centro di questo giudizio vi è dunque solo, ed esclusivamente, ciò che è stato convenuto e co-deliberato circa il patto di governo che è anche atto di conferimento del potere governante.

La scomposizione della semantica giuridico-politica compiuta sul testo del Dotzè eiximeniano consente di mettere in evidenza alcuni elementi dottrinari decisivi.

In primo luogo la sede, il contesto in cui il passo citato viene a collocarsi nell'opera politica scritta dal frate minore.

L'autorità politica viene delineata, discussa nella sua origine, analizzata nei suoi fondamenti di legittimazione ed autorevolezza, all’interno dei capitoli dedicati a specificare quale sia la quinta ratio per la quale la città si costituisce, vale a dire quella ragione di natura prettamente economica che ha i suoi fondamenti primi nel passo della Politica in cui si definisce la polis come luogo in cui si realizza la sufficientia vitae. Un passo che trova una riformulazione eiximeniana che parte dalla definizione della ciutat come comunità politica e civile grazie alla quale gli uomini trovano la soddisfazione sufficiente ai propri bisogni potendo "satistfer e provehir 
bestantment" ad essi. Si tratta di una definizione che registrerà un'amplificazione particolarmente significativa in quanto, in questa stessa opera, essa verrà riletta ed approfondita dal Francescano sino a divenire un vero e proprio quadro di analisi economica, finanziaria e specificamente monetaria occupando decine di capitoli, sempre collocati nella sezione dedicata ad analizzare l'origine e gli obiettivi della polis.

Che la definizione dell'autorità politica e delle forme con le quali essa diviene legittimo governante stia all'inizio di questo percorso ha quindi un'evidente coerenza logica e dottrinaria che non è solo formale: il Gerundense infatti fa della dimensione economica, della ragione degli scambi e del mercato il tratto costitutivo principale della e delle comunità politiche. Egli individua, a partire dalla definizione aristotelica della comunità come sede delle relazioni comunicative (communicatio) e degli scambi (commutatio), il vero motore, il vero elemento coesivo e dinamico che costituisce e dà forza alla civitas ${ }^{8}$. In questa dimensione l'autorità politica acquista un suo forte elemento di validazione non solo nel quadro giuridico e politico che abbiamo letto attraverso la descrizione del primo re legittimo, ma in quanto questa autorità consente e favorisce la realizzazione della vita buona, la vita adeguata $\mathrm{e}$ comunicativa degli scambi che fa la civitas, che è la sua ragion d'essere. E' precisamente ciò che Eiximenis definisce e qualifica come civilitas.

Nel nesso tra l'economico ed il civile consiste una delle più rilevanti differenze che contraddistinguono il discorso eiximeniano sull'origine e la legittimazione del potere e quanto viene sostenuto in quello stesso secolo, prima del testo che stiamo analizzando, in particolare da Marsilio. E' infatti leggendo il Defensor pacis e il

\footnotetext{
${ }^{8 " T u t t e ~ l e ~ m e r c i ~ d e v o n o ~ e s s e r e ~ v a l u t a t e ~ i n ~ m o n e t a: ~ c o s i ̀ ~ i n f a t t i ~ s a r a ̀ ~ s e m p r e ~ p o s s i b i l e ~ u n o ~ s c a m b i o, ~ e, ~ s e ~ s a r a ̀ ~ p o s s i b i l e ~ l o ~ s c a m b i o, ~ s a r a ̀ ~}$ possibile anche la comunità ... infatti non ci sarebbe comunità senza scambio" (ARISTOTELES LATINUS, Et. Nic. V,5 1133b 15-18). Pochi passi più sopra Aristotele aveva stabilito il nesso profondo tra legge-istituzione e comunità come luogo della comunicazione, definita Koıvwvía di scambio reso possibile dalla moneta. Lì egli sosteneva che "la moneta, che consente la comunicazione dello scambio è nata per convenzione, e per questo", dunque per questa unità pre-costituita tra i soggetti politici ed economici dello scambio, "ha il nome di nomisma, perché non esiste per natura ma per nomos, (perché non è sorta "secondo natura", ma "secondo istituzione") e perché dipende da noi cambiarne il valore o renderla inutile", vale a dire dipende da noi la deliberazione che la trasforma o la rende inutilizzabile (ARISTOTELES LATINUS,Et. Nic. V,5 1133a 29-31).
} 
Defensor minor che si notano con maggior evidenza le differenze con Eiximenis9 9 Se entrambi pongono la dimensione della comunità politica esemplificata nella polis al centro del loro sistema, divenendo essa la chiave strutturale e lo strumento finale per la realizzazione della politicità e della civiltà dell'uomo, emerge con chiarezza come in Marsilio l'assenza di riflessione sulla dimensione civile dell'economia, sulla ragion d'essere degli scambi, la mancanza di ogni riflessione sull'istituzione e sul senso della moneta, costituiscano i maggiori elementi che differenziano il discorso sul potere svolto dal Padovano da quello messo in forma dal Gerundense.

Se confrontiamo invece le riflessione del Frate catalano con la quaestio di Scoto potremo considerare la posizione di Eiximenis sulla legittimazione del governante ed il suo inserimento funzionale nella quinta ratio esplicativa dell'origine e delle finalità della polis come un importante sviluppo della definizione del buon legislator offertaci dal Doctor Subtilis. Si ricordi che il francescano inglese sosteneva che il legislatore può dirsi buon legislatore, e dunque adeguato ai suoi compiti, se è capace di analizzare la realtà economica della res publica che è chiamato a governare, per consenso e deliberazione comunitaria, e se è capace di individuare le misure più idonee, compensi compresi, per favorire l'attività e la professione dei mercanti nella e per la res publica.

La collocazione della riflessione eiximeniana sul buon e legittimo governante nella sua discussione intorno alla ratio economica, fondativa della polis, e soprattutto il fatto che egli dia poi corpo e sostanza ad una valorizzazione civile, politica ed economica della figura del mercator, capace di dinamizzare gli scambi e le risorse monetate quale esempio di massima comprensione del senso e del valore della civitas, sono elementi sufficienti per proporre una comparazione tra la sua analisi del potere legittimo e quanto ebbe ad insegnare Scoto ai suoi allievi nelle sedi delle accademie parigina ed oxoniense.

\footnotetext{
${ }^{9}$ Sulla vita buona in Marsilio, si vedrà utilmente un testo importante di Gianluca Briguglia (BRIGUGLIA, 2013, p. 75- 84). La vita buona, secondo l'impostazione strettamente aristotelica, è tale perché funzionale alle predisposizioni razionali dell'uomo e si realizza nella polis grazie all'ordinamento politico, ordinamento che ha per scopo immediato proprio quello di sostenere gli uomini nella loro ragionevolezza e nel realizzare, appunto, il loro bene vivere. Lo rileva con grande chiarezza Jurgen Miethke (MIETHKE, 2005, p. 237).
} 
La figura di Forseo, legittimato al potere dal consenso e dal patto e la figura del primo re di Tessaglia evocato in quello stesso capitolo come immagine chiave del buon governante in quanto re che ha coniato la prima moneta d'oro e per primo ha migliorato l'arte che rendeva possibili ed efficienti gli scambi, hanno così un evidente valore retorico che va inteso nel senso più pieno del termine. La retorica politica, infatti, soprattutto in un'età ove non esiste una sistematizzazione disciplinare dei saperi, riveste un valore dottrinario, pedagogico e formativo che non può essere celato dietro una considerazione della retorica come mera forma, cifra stilistica del linguaggio adottato dallo scrivente o dall'oratore.

L'analitica descrizione delle modalità con le quali si conferisce il potere al governante che si è letta più sopra a proposito di Forseo va quindi colta nella sua pienezza e nella sua complessità a partire da alcuni elementi chiave. Si tratta di elementi che servono a comprendere meglio il valore strettamente politologico delle posizioni del Gerundense assunte rispetto ad un nodo centrale della teoria politica.

Come una solida storiografia ha giustamente messo in luce, è infatti evidente che il consenso sia un elemento ben presente nella riflessione politica e giuridica tardomedievale, quantomeno in alcune aree culturali e politiche dell'Europa ${ }^{10}$. Ma nei testi francescani che si sono utilizzati il consenso acquisisce uno statuto di tutt'altra pregnanza, innanzitutto perché non è più una manifestazione contingente, scambiabile con la nozione di assenso o legata all'approvazione di una singola norma: esso diviene prerequisito fondante del costituirsi stesso del potere, essendo in Scoto parametro esclusivo della sua legittimazione. Se osserviamo poi il testo del suo allievo si vede come il governante può costituirsi come autorità legittima sulla base di un consenso che non è semplicemente manifestato, ma è conferito e revocabile, è - nelle pagine eiximeniane - dotato di una sua forma e di contenuti propri, ciascuno dei quali considerato come parte indefettibile del consenso pattizio stesso, pena la sua invalidità. Nel capitolo decisivo del frate catalano dedicato alla

${ }^{10} \mathrm{Nell}$ 'ambito di una storiografia sul tema davvero assai vasta si segnala, tra i contributi recenti più importanti, il saggio di Carl Nederman (NEDERMAN, 2009, p. 63-138). 
fondazione dell'autorità politica la stessa revocazione del potere non si limita all'enunciazione del principio, ma diviene procedura giuridica e politica. Revoca del potere non significa solo affermazione di un principio astratto o possibilità teorica di una destituzione violenta, né tantomeno giustificazione del tirannicidio. In Eiximenis la revoca del potere si concretizza in istituto giuridico e procedurale che, essendo precostituito, offre un alveo di garanzie ad entrambi i soggetti politici coinvolti: governante e governati. Essa è quindi una procedura di natura garantista che si rivela tale non solo in questo passo fondamentale del costituirsi del potere e dell'autorità, ma anche nei casi, ben definiti, in cui il governante viene riconosciuto colpevole di reati in danno della comunità che integrano una laesio dell'intero corpo politico, significativamente concretizzati in due crimini di piena valenza economica: il furto e l'alterazione di qualsiasi parametro della moneta, considerata un'istituzione che appartiene non al coniatore ma alla comunità che la usa e vi si identifica (EIXIMENIS, 2005, capp. 58 e 139-141, p. 126- 127 e p. 303-308).

Vi è inoltre un ulteriore elemento che consente di avvicinare pienamente la dottrina politica eiximeniana che analizza l'origine del potere e quella prodotta dal suo maestro inglese.

Si tratta della questione della forma istituzionale del potere.

Se per il Doctor Subtilis, ma anche per il Doctor Invincibilis, l'autorità politica viene legittimata ed è considerata valida al di là di ogni valutazione circa la veste istituzionale che essa assume, escludendo in particolare la legittimazione fondata sulla sola forma di governo, in Eiximenis la questione è posta in maniera solo parzialmente diversa ${ }^{11}$.

Se da un lato nel Dotzè i termini di re, princeps e governante sono spesso intercambiabili, proprio in quanto a prevalere è la funzione del governo su quella

\footnotetext{
${ }^{11}$ Per Ockham si legga in particolare la sua duplice riflessione sulla legittimità del potere contenuta nel Dialogus de potestatae pape et imperatoris: "L'utilità comune è la causa per cui un unico sommo pontefice deve essere posto a capo di tutti i fedeli. Allora se dal governo di uno solo non deriva l'utilità comune ma il danno comune questa forma di governo deve cessare immediatamente. Allora la comunità dei fedeli deve avere il potere di istituire immediatamente un'altra forma di governo" (III, I, ii, cap. 20). "L'imperatore non detiene la pienezza del potere in ambito temporale ... ma il suo potere è limitato, perché può compiere, riguardo agli uomini liberi suoi sudditi e ai loro beni, solo quanto si giustifica in vista dell'utilità comune" (III, II, ii, cap. 27), (OCKHAM, 1999, p. 181 e p. 183, corsivi nostri).
} 
della figura di chi la esercita, vi è però un dato non nominalistico, ma di grande peso che emerge con nettezza nel pensiero del Catalano. Il reggitore - sostiene Eiximenis - può assumere qualsiasi denominazione, ma non può mai fregiarsi del titolo, del nome di senyor.

A conferma di quale sia il vero oggetto di riflessione del frate quando discute dell'essenza del potere e del governo egli sostiene che ai principes non è concessa altra forma di potere "sinó pura aministració e juredicció paccionada, segon que el començament de lur regiment estech per lo poble atorgada a ells o a lurs predecessors per millor estament de la cosa pública e per conservació de pau e de justícia en lo poble qui.ls elegia". Da ciò ne discende che "jamés en lo començament, quant se faeren eleccions dels prínceps, no.ls estech atorgada senyoria sinó ço que dit és, e aquesta fo la rahó per què los regens major foren apellats reys, ço és regens los altres, e foren appellats prínceps, ço és hòmens principals en lo poble, o presidens, ço és aquells qui van davant los altres; mas jamés lo poble no.ls atorgà nom de senyoria, per tal que no passàs lur regiment en tirannia, e per tal que no cuydassen haver plena senyoria ..." (EIXIMENIS, 1986, cap. 645, p. 434, corsivi nostri).

Per il francescano allievo di Scoto la plenitudo potestatis, la pienezza del potere è quindi intrinsecamente incompatibile con la concezione del potere legittimo.

\section{Conclusione}

La precisione con la quale Eiximenis si esprime sull'autorità politica considerata sotto il profilo della sua legittimità va registrata in via conclusiva per rilevare come alcuni capitoli della sua opera politica, che presenta ancora la veste formale di uno speculum principis, o comunque le sembianze di un trattato di etica politica, consegua al suo interno livelli di analisi e di definizione dottrinaria sulla questione del potere di notevole peso dimostrando una matrice comune condivisa con i livelli più alti raggiunti dalla testualità politica francescana nel secolo XIV. Si 
pensi, ancora una volta, alle tesi di Scoto e a quelle, in questa sede appena accennate, di Ockham.

Da questo punto di vista gli sviluppi raggiunti dal frate catalano dimostrano come quella linea di analisi pauperista che muove le sue riflessioni a partire dall'origine del dominium definita come facoltà concessa dal Creatore, ma interamente consegnata alla libera volizione dell'uomo postlapsario, messa in forma dai filosofi francescani nella prima metà del '300, mantenga una sua indubbia fecondità lungo tutto quel secolo, costituisca in effetti un autentico filo rosso del pensiero politico prodotto dai Minori secondo una chiave ermeneutica assai ben definita ed utilizzata negli studi di Roberto Lambertini dedicati appunto alla “povertà pensata” (LAMBERTINI, 2000, p. 51-79, p. 189- 288).

L'esito politico e politologico di questa riflessione francescana scaturisce infatti da una necessità e da una contingenza storica che non possono essere sottostimate: è l'Ordine dei Minori, messo sotto accusa da chi sostiene l’impraticabilità del vivere in volontaria povertà, a porre la questione su un piano più alto. Gli esponenti dell'Ordine vogliono dimostrare come il diritto a possedere, ancorché concesso a Dio a tutti gli uomini discendenti di Adamo, non è un dovere, ma una facoltà, giustificando così il diritto stesso all'esistenza dell'Ordo Minorum. Questa dimostrazione, necessitata storicamente dalla congiuntura nella quale i Minori si trovano a vivere e a tutelare la loro identità, non si limita ad ottenere quel pur importante e primario risultato, essa porta con sé la conquista di nuovi spazi di analisi e nuovi contenuti teorici di natura squisitamente filosofica e politica, spazi e contenuti che ineriscono alla libertà, alla volontà dell'uomo ed alla legittimazione del potere che quest'uomo ha così il diritto di scegliersi.

\section{RIFERIMENTI BIBLIOGRAFICI}

AGAMBEN, Giorgio. De la très haute pauvreté. Règles et forme de vie. Paris: Payot \& Rivages, 2011.

AGAMBEN, Giorgio. Il regno e la gloria.Per una genealogia teologica dell'economia e del governo. Vicenza: Neri Pozza, 2007. 
ARISTOTELES LATINUS. Ethica Nicomachea, translatio Roberti Grosseteste

Lincolniensis, B. recensio recognita. Leiden - Bruxelles: Brill, 1973.

ASSMANN, Jan. Herrschaft und Heil. Politische theologie in Altägypten, Israel und Europa. München - Wien: Hanser, 2000.

BRIGUGLIA, Gianluca. Marsilio da Padova. Roma: Carocci, 2013.

CAPITANI, Ovidio. Tradizione ed interpretazione: dialettiche ecclesiologiche del secolo XI. Roma: Jouvence, 1990.

COSTA, Pietro. Civitas. Storia della cittadinanza in Europa, I. Roma-Bari: Laterza, 1999.

DOLCINI, Carlo. Crisi di poteri e politologia in crisi: Da Sinibaldo de Fieschi a Guglielmo d'Ockham. Bologna: Pàtron, 1988.

DUNS SCOTO, Johannes. Ordinatio Oxoniensis IV, d. 15, q. 2. In: A. B. Wolter. Duns Scotus....Saint Bonaventure (N. Y.): The Franciscan Institute, 2001.

EIXIMENIS, Francesc. Dotzè Llibre del Crestià, I, 1. Girona: Universitat de Girona Diputació de Girona, 2005.

EIXIMENIS, Francesc. Dotzè Llibre del Crestià, II.1. Girona:Collegi Universitari de Girona - Diputació de Girona, 1986.

ESPOSITO, Roberto. Due. La macchina della teologia politica e il posto del pensiero. Torino: Einaudi, 2013.

EVANGELISTI, Paolo. Ad invicem participancium. Un modello di cittadinanza proposto da Francesc Eiximenis, frate francescano. Mélanges de l'École française de Rome - Moyen Âge,Roma, n. 125-2, 2013. Disponibile il: <http://mefrm.revues.org/1466>. Accesso il : 12 dic. 2017.

EVANGELISTI, Paolo. I francescani e la costruzione di uno Stato. Linguaggi politici, valori identitari, progetti di governo in area catalano-aragonese, Padova: EBF, 2006.

EVANGELISTI, Paolo. Relazioni di potere ed etiche per il potere. Clareno, Filippo di Maiorca e la testualità politica francescana catalano-aragonese. CLARENO, Angelo Atti del XXXIV Convegno internazionale, Assisi, 5-7 ottobre 2006, Spoleto, 2007. p. 317-376.

LAMBERTINI, Roberto. La povertà pensata. Modena: Mucchi, 2000.

LENOBLE, Clement, L'exercice de la pauvreté. Économie et religion chez les franciscains d'Avignon (XIII ${ }^{\mathrm{e}} \mathrm{XV}^{\mathrm{e}}$ siècle), Rennes: PUR, 2013.

LENOBLE, Clement; TONEATTO, Valentina. La pauvreté comme acte de résistance?. La vie des idees.fr, 18 septembre 2013. Disponilile il: <http://www.laviedesidees.fr/La-pauvretecomme-acte-de-resistance.html>. Accesso il: 12 dic. 2017.

MANCINELLI, Chiara. Teoria e pratica economica francescana. Il convento del Santo Spirito del Monte (Gilet, Valencia). Roma: Aracne, 2017. 
MARSILIO DA PADOVA.Defensor pacis, ed. C. Vasoli. Torino: Utet, 1975.

MIETHKE, Jurgen. Ai confini del potere. Il dibattito sulla potestas papale da Tommaso d'Aquino a Guglielmo d'Ockham. Padova: EBF, 2005.

MINEO, E. Igor. Caritas e bene comune. Storica, Milano, ano 20, p. 7- 56, 2004.

NEDERMAN, Carl. Lineages of European Political Thought. Washington D.C.: The Catholic University of America Press, 2009.

OCKHAM, Guglielmo. Breviloquium (Opera politica, IV). Ed. H. S. Offler. Oxford: Oxford Univeristy Press 1997.

OCKHAM, Guglielmo. Dialogus de potestate papae et imperatoris. In: ANDREATTA; Alberto; MARTIGNETTI, Giuliano. Il pensiero politico. Idee teorie dottrine, Antologia.Trad. M. Conetti.Eds. A. Andreatta, A. E. Baldini, C. Dolcini. Torino: UTET, 1999, IV. I.

OLIVI, Pietro Di Giovanni. Quid ponat ius vel dominium. Antonianum, Roma, 20, p. 309$330,1945$.

SCHMITT, Carl. La tirannia dei valori. Milano: Adelphi, 2009.

SCHMITT, Carl. Le categorie del "politico", eds. G. Miglio - P. Schiera. Bologna: Il Mulino, 1972.

TODESCHINI, Giacomo. Un trattato di economia politica francescana: il De emptionibus et venditionibus, de usuris, de restitutionibus di Pietro di Giovanni Olivi. Roma: ISIME,1980.

TODESCHINI, Giacomo. Il prezzo della salvezza. Lessici medievali del pensiero economico. Roma: Nuova Italia Scientifica, 1994.

TODESCHINI, Giacomo. I mercanti e il tempio. La società cristiana e il circolo virtuoso della ricchezza fra Medioevo ed età moderna. Bologna: Il Mulino, 2002.

TODESCHINI, Giacomo. Visibilmente crudeli. Malviventi, persone sospette e gente qualunque dal Medioevo all'età moderna. Bologna: Il Mulino, 2007.

TODESCHINI, Giacomo. Come Giuda. La gente comune e i giochi dell'economia all'inizio dell'epoca moderna. Bologna: Il Mulino, 2011.

TODESCHINI, Giacomo. I diritti di cittadinanza degli ebrei italiani nel discorso dottrinale degli Osservanti. In: I frati osservanti e la società in Italia nel secolo XIV, Atti del XL convegno internazionale, Assisi - Perugia, 11 - 13 ottobre 2012. Spoleto: CISAM, 2013, p. 254- 277.

TONEATTO, Valentina. Marchands et banquiers du Seigneur. Lexiques chrétiens de la richesse et de l'administration monastiques de la fin du $\mathrm{IV}^{\mathrm{e}}$ siècle au début du $\mathrm{IX}^{\mathrm{e}}$ siècle. Rennes: Presses Universitaires de Rennes, 2012. 